\title{
Sliding Mode Controller for Satellite Attitude Stabilization Maneuver
}

\author{
Licheng Feng $^{1, a}$, Xiaoqian Chen ${ }^{1, b}$, Tao Sheng ${ }^{1, c}$ \\ ${ }^{1}$ National University of Defense Technology, Changsha 410073, China; \\ aflch1992@163.com, 'bchenxiaoqian@nudt.edu.cn, 'shengtao-2002@163.com
}

Keywords: Sliding mode control, rigid satellite, attitude control, Lyapunov method

\begin{abstract}
Attitude control system of the rigid satellite is a coupling and uncertain nonlinear system with multi-inputs and multi-outputs. This paper presents a sliding mode controller for the satellite attitude system with limited control input and external disturbances. The design strategy can guarantees that the closed-system is global asymptotic stable and realizes the requirements of the control input saturation. The control algorithm is model-independent and it performs effectively on restraining bounded external disturbances.
\end{abstract}

\section{Introduction}

Satellite motion can be described by the kinematic and dynamic equations [1, and 5]. In practice, the mathematical description of satellite motion is highly nonlinear and coupling, thus the conventional linear control technique is not suitable for the controller design. In order to realizing satellite's stabilization, the attitude control system must take these nonlinearities into consideration.

Sliding Mode Control (SMC) has been recognized as one of the efficient methods for the coupling and uncertain nonlinear system [3, 6 and 7]. The main advantages of SMC are its fast dynamic response, robustness, simplicity in design and implementation. The disadvantage of SMC is the chattering phenomenon, which could be avoided by adopting the boundary layer technique [8, and 9].

This paper presents a control strategy which based on the sliding mode control for the rigid satellite attitude maneuver. The structure of the paper is organized as follows. Preliminaries are given in section 2. In section 3, a control law is proposed and related convergence analyses are also provided. Section 4 and section 5 give the numerical simulation and conclusion respectively.

\section{Preliminaries}

\subsection{Kinematic Equation}

In this paper, the quaternion is adopted to describe the attitude of a rigid satellite for its global representation without singularity. The quaternion is defined by $\boldsymbol{Q}=\left[q_{0}, \boldsymbol{q}^{T}\right]^{T}$ and it is subject to

$$
q_{0}^{2}+\boldsymbol{q}^{T} \boldsymbol{q}=1
$$

The kinematic equation [2] in terms of the unit quaternion is given by:

$$
\begin{aligned}
& \dot{\boldsymbol{q}}=\frac{1}{2}\left(q_{0} \boldsymbol{I}_{3}+\boldsymbol{q}^{\times}\right) \omega \\
& \dot{q}_{0}=-\frac{1}{2} \boldsymbol{q}^{T} \boldsymbol{\omega}
\end{aligned}
$$

Where $\boldsymbol{I}_{3}$ denotes the identity matrix and $\boldsymbol{\omega}$ is the body angular velocity vector represented in body frame with respect to the inertial frame. The notation $\boldsymbol{q}^{\times}$is a skew-symmetric matrix expressed by:

\subsection{Dynamic Equation}

$$
\boldsymbol{q}^{\times}=\left[\begin{array}{ccc}
0 & -q_{3} & q_{2} \\
q_{3} & 0 & -q_{1} \\
-q_{2} & q_{1} & 0
\end{array}\right]
$$

The dynamic equation of a rigid satellite is given by [2]: 


$$
J \dot{\omega}+\omega^{\times} J \omega=u(t)+d(t)
$$

Where $\boldsymbol{J} \in R^{3 \times 3}$ denotes the inertia tensor of the satellite, $\boldsymbol{u}=\left[u_{1}, u_{2}, u_{3}\right]^{T}$ is the control torque, and $\boldsymbol{d}=\left[d_{1}, d_{2}, d_{3}\right]^{T}$ represents the bounded external disturbances, where $\left|d_{i}\right| \leq \delta_{i}, i=1,2,3$ and $\delta_{i}$ is a positive constant.

\subsection{Control objective}

During the whole attitude maneuver, some assumptions are made.

\section{Assumption 1}

In satellite model equations, the unit quaternion $\boldsymbol{q}$ and the body angular velocity $\boldsymbol{\omega}$ are available in the feedback control design.

\section{Assumption 2}

The external disturbances $\boldsymbol{d}$ come from the gravitational perturbation, solar radiation pressure, electromagnetic force, etc. are bounded.

\section{Assumption 3}

Each component of the control torque $\boldsymbol{u}$ is constrained by a bounded value, and expressed by

$$
\left|u_{i}(t)\right| \leq u_{\max }, \forall t>0, i=1,2,3
$$

The attitude control problem in this paper is to design the control law $\boldsymbol{u}$ such that:

(a) The closed-loop system is globally stable in that all the signals are bounded and continuous;

(b) The quaternion $\boldsymbol{q}$ and the angular velocity $\boldsymbol{\omega}$ converge to an arbitrary small set in finite time, which is $\|\boldsymbol{q}(t)\| \leq \varepsilon_{1}$ and $\|\boldsymbol{\omega}(t)\| \leq \varepsilon_{2}$.

\section{Controller design}

The main idea of the SMC is to design a high-speed control algorithm which can drive the state trajectory of the nonlinear system onto a sliding surface during finite time and maintain the state trajectory on the sliding surface [1]. Hence, the selection of a sliding surface and the design of the control law are the main two steps. In this section, a sliding mode controller based on unit quaternion attitude representation is developed.

\subsection{Sliding surface design}

A new notation is introduced for simplicity of expression

$$
\operatorname{sig}(x)^{\alpha}=|x|^{\alpha} \operatorname{sgn}(x), 0<\alpha<1
$$

It should be noticed that once $0<\alpha<1, \operatorname{sig}(x)^{\alpha}$ is continuous but not differentiable.

A sliding surface [3, 4 and 5] in vector form is defined as follows:

$$
\boldsymbol{s}=\boldsymbol{\omega}+\boldsymbol{C} \operatorname{sig}(\boldsymbol{q})^{\alpha}
$$

Where $\boldsymbol{C}=\operatorname{diag}\left(c_{1}, c_{2}, c_{3}\right)$ and $c_{i}>0$.

Once the state trajectory of the satellite attitude system is on the sliding surface, i.e. $\boldsymbol{s}=\mathbf{0}$, it follows

$$
\boldsymbol{\omega}=-\boldsymbol{C} \operatorname{sgn}(\boldsymbol{q})^{\alpha}
$$

Now, considering the Lyapunov function candidate

$$
V_{1}=\left(1-q_{0}\right)^{2}+\boldsymbol{q}^{T} \boldsymbol{q}
$$

With equation (2) and equation (8), the time derivative of $V_{1}$ can be calculated as

$$
\dot{V}_{1}=-2\left(1-q_{0}\right) \dot{q}_{0}+2 q^{T} \dot{q}=-\sum_{i=1}^{3} c_{i}\left|q_{i}\right|^{\alpha+1}
$$

Equation (10) implies that $\dot{V}_{1}=$ 0if and only if $\boldsymbol{q}=\mathbf{0}$. Therefore, $V_{1}$ is a Lyapunov function such that the quaternion $\boldsymbol{q}$ will converge to zero and $q_{0}$ tends to \pm 1 when $t \rightarrow \infty$ according to equation (1). 
It should be noted that the equilibrium point $\left(q_{0}, \boldsymbol{q}\right)=(-1, \mathbf{0})$ is not stable. To prove this, the following Lyapunov function is considered.

The derivative is

$$
V_{2}=\left(1+q_{0}\right)^{2}+\boldsymbol{q}^{T} \boldsymbol{q}
$$

$$
\dot{V}_{2}=2\left(1+q_{0}\right) \dot{q}_{0}+2 q^{T} \dot{q}=\sum_{i=1}^{3} c_{i}\left|q_{i}\right|^{\alpha+1}
$$

From equation (12), it is obvious that $\left(q_{0}, \boldsymbol{q}\right)=(-1, \mathbf{0})$ is not a stable equilibrium point.

\subsection{Control law design}

The sliding mode control law [3] divided into two parts:

$$
\boldsymbol{u}(t)=\boldsymbol{u}_{e q}+\boldsymbol{u}_{s w}
$$

$\boldsymbol{u}_{e q}$ will make sliding surface invariant and it is computed by setting $\dot{\boldsymbol{s}}=0$ considering $\boldsymbol{s}=\boldsymbol{0} . \boldsymbol{u}_{s w}$ is an extra control which forces the quaternion and the angular velocity to reach on sliding surface during finite time.

To obtain $\boldsymbol{u}_{e q}$, we set $\dot{\boldsymbol{s}}=\mathbf{0}$ and combine equation (7), equation (2) and equation (4) and we can get

$$
\frac{1}{J}\left(-\boldsymbol{\omega}^{\times} \boldsymbol{J} \boldsymbol{\omega}+\boldsymbol{u}(t)+\boldsymbol{d}(t)\right)=-\frac{\alpha}{2} \boldsymbol{C d i a g}\left(\left|q_{i}\right|^{\alpha-1}\right)\left(q_{0} \boldsymbol{I}_{3}+\boldsymbol{q}^{\times}\right) \boldsymbol{\omega}
$$

According to equation (14), $\boldsymbol{u}_{e q}$ can be chosen as

$$
\boldsymbol{u}_{e q}(t)=\boldsymbol{\omega}^{\times} \boldsymbol{J} \boldsymbol{\omega}-\boldsymbol{d}_{c}(t)-\frac{\alpha}{2} \boldsymbol{J C d i a g}\left(\left|q_{i}\right|^{\alpha-1}\right)\left(q_{0} \boldsymbol{I}_{3}+\boldsymbol{q}^{\times}\right) \boldsymbol{\omega}
$$

Where $\boldsymbol{d}_{c}$ satisfies $\left|d_{c i}\right| \geq\left|d_{i}\right|[3], i=1,2,3$

The SMC reaching law [3] is selected

Where $k_{\mathrm{i}}>0$ and $0<\beta<1$.

$$
\dot{s}_{i}=-k_{i}\left|s_{i}\right|^{i} \operatorname{sgn}\left(s_{i}\right)
$$

$\boldsymbol{u}_{s w}$ is calculated according to the reaching law (16) as

Therefore, the designed control law is

$$
\mathbf{u}_{s w}=-\boldsymbol{K} \operatorname{sig}(s)^{\beta}
$$

$$
\boldsymbol{u}(t)=\boldsymbol{\omega}^{\times} \boldsymbol{J} \boldsymbol{\omega}-\boldsymbol{d}_{c}-\boldsymbol{K} \operatorname{diag}\left(\left|s_{i}\right|^{\beta}\right) \operatorname{sgn}(s)-\frac{\alpha}{2} \boldsymbol{J C d i a g}\left(\left|q_{i}\right|^{\alpha-1}\right)\left(q_{0} \boldsymbol{I}_{3}+\boldsymbol{q}^{\times}\right) \boldsymbol{\omega}
$$

Now, considering the following Lyapunov function candidate

$$
V_{3}=\frac{1}{2} \boldsymbol{s}^{T} \boldsymbol{J} \boldsymbol{s}
$$

Differentiating $V_{3}$ with respect to time and obtaining the following expression

$$
\dot{V}_{3}=\boldsymbol{s}^{T} \boldsymbol{J} \dot{\boldsymbol{s}}=\boldsymbol{s}^{T}\left[-\boldsymbol{\omega}^{\times} \boldsymbol{J} \boldsymbol{\omega}+\boldsymbol{u}+\boldsymbol{d}+\frac{\alpha}{2} \boldsymbol{J} \boldsymbol{C} \operatorname{diag}\left(\left|q_{i}\right|^{\alpha-1}\right)\left(q_{0} \boldsymbol{I}_{3}+\boldsymbol{q}^{\times}\right) \boldsymbol{\omega}\right]
$$

By substituting equation (18) into equation (20), the derivation of $V_{3}$ can be simplified as

$$
\dot{V}_{3}=\boldsymbol{s}^{T}\left(-\boldsymbol{K} \operatorname{sig}(s)^{\beta}+\boldsymbol{d}(t)-\boldsymbol{d}_{c}(t)\right)
$$

This is clearly negative definite provided selecting appropriate $\boldsymbol{d}_{c}$.

\subsection{Chattering avoidance}

In order to avoid the chattering phenomenon which may cause possible damages to the actuators, boundary layer method is introduced to reduce the chattering. The basic idea is to replace the sign function with a saturation function [3] which defined as 


$$
\operatorname{sat}(s)=\left\{\begin{array}{cc}
1 & s>\Delta \\
s / \Delta & |s| \leq \Delta \\
-1 & s<-\Delta
\end{array}\right.
$$

Where $\Delta$ is the boundary layer. Normally the larger the boundary layer width, the smoother the control signal. However, it no longer drives the system to the origin, but to within the chosen boundary layer instead. Very small thickness layer can also introduce the chattering phenomena.

\section{Simulation results}

To verify the effectiveness and the performance of the proposed control law, numerical simulations have been carried out using the rigid satellite system of equation (2) and (4) together with the developed sliding mode control law of equation(18). Considering a rigid satellite with the nominal inertia matrix $\boldsymbol{J}=\operatorname{diag}(20,21,22) \mathrm{kg} \cdot \mathrm{m}^{2}$. The initial attitude orientation of the unit-quaternion is $\boldsymbol{Q}=[0.97601,-0.070428,0.10058,-0.17981]^{T}$ (equivalent to the initial Euler angles $\left.\left[-10^{\circ}, 10^{\circ}, 20^{\circ}\right]^{T}\right)$. The initial body angular velocity is $\omega(0)=[0.12,-0.15,0.11]^{T} \mathrm{rad} \cdot \mathrm{s}^{-1}$. The external disturbance is $\boldsymbol{d}(t)=\sin (0.5 \mathrm{t})[0.004,-0.003,0.007]^{T} N \cdot m$. The desired quaternion and body angular velocity is $\boldsymbol{Q}_{d}=[1,0,0,0]^{T}$ and $\boldsymbol{\omega}_{d}=[0,0,0]^{T} \mathrm{rad} \cdot \mathrm{s}^{-1}$ respectively.

The parameters related to the controller are chosen as $\alpha=0.85, \beta=0.9, \boldsymbol{K}=\operatorname{diag}(18,18,18)$, $\boldsymbol{C}=\operatorname{diag}(0.4,0.4,0.4)$ and $\boldsymbol{d}_{c}=[0.01,0.01,0.01]^{T} N \cdot m$.

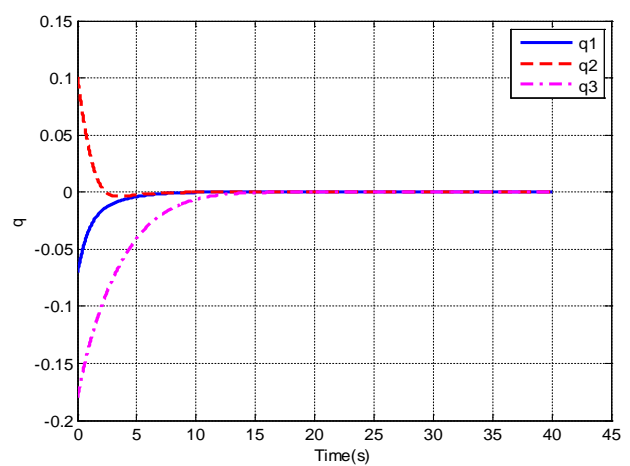

Fig. 1 Response of the quaternion

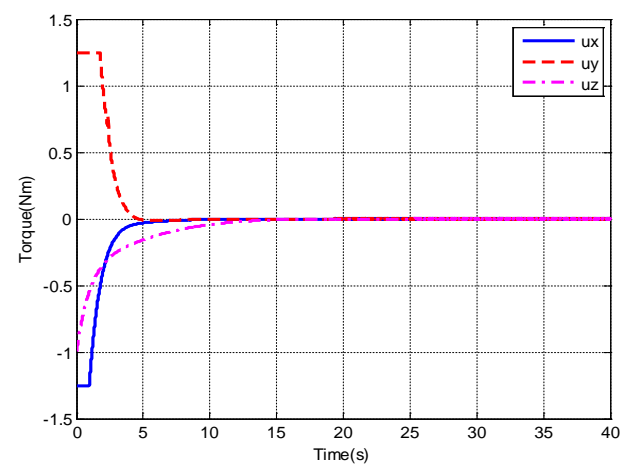

Fig. 3 Response of the control input

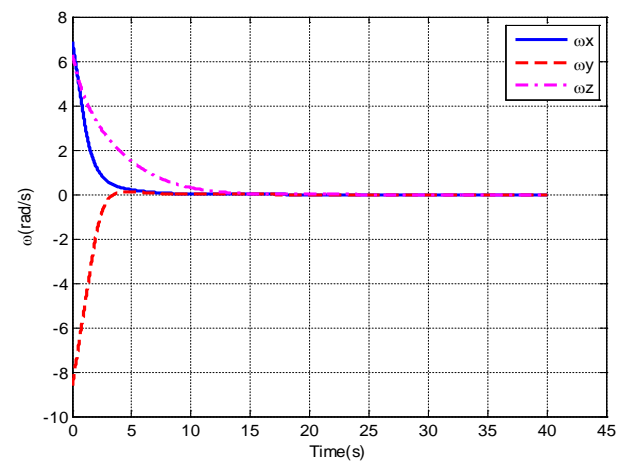

Fig. 2 Response of attitude velocity

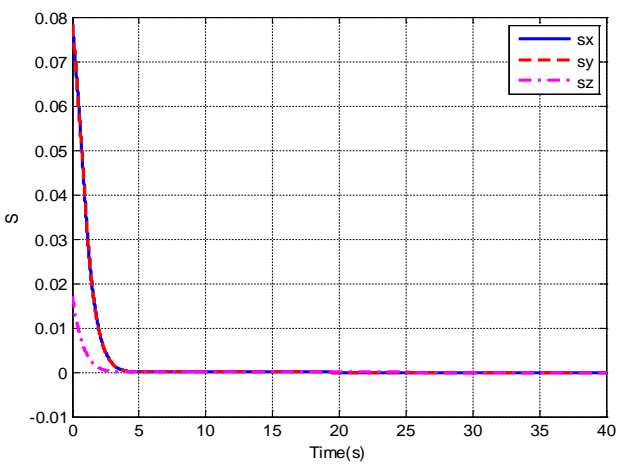

Fig. 4 Response of sliding surface function

Simulation results are presented in Fig. 1-Fig. 4. Fig. 1 illustrates the behavior of the attitude quaternion; Fig. 2 presents the response of the body angular velocity; Fig. 3 is the time history of the control torque and Fig. 4 shows the behavior of the sliding surface function. During the simulation, we bound the magnitude of the torque as $\left|u_{i}\right| \leq 1.25$. 


\section{Conclusion}

In this study, a sliding mode controller is proposed for the rigid satellite in the presence of external disturbances and limited control input. The proposed controller can drive the rotational motion of the rigid satellite to its desired trajectory in finite time. In the whole process, the attitude variables achieved are shown to be global asymptotically stable through the standard Lyapunov technique. Moreover, the proposed control strategy can guarantee no chattering occurrence and the confirmation of the designed controller parameters is simple. Numerical simulations also demonstrate the effectiveness and performance of the proposed control scheme.

\section{References}

[1] James R. Wertz, "Spacecraft Attitude Determination and Control”, Academic Publisher, Dordrecht, Boston, London, 1991.

[2] Huang Zhengui, Attitude Dynamics of Spacecraft, first ed., Chang Sha, 1997.

[3] Liu Jinkun, SLIDING MODE CONTROL DESIGN AND MATLAB SIMULATION, second ed., Beijing, 2012.

[4] Azza EI-S. Ibrahim, Ahamed M. Tobal, Mohammad A.Sultan, Satellite Attitude Maneuver using Sliding Mode Control under Body Angular Velocity Constraints, International Journal of Computer Applications,Vol.50, No.13, 0975-8887, July 2012.

[5] Jin Erdong, Sun Zhaowei, Robust controllers design with finite time convergence for rigid spacecraft attitude tracking control, Aerospace Science and Technology 12 (2008) 324-330.

[6] A. Bartoszewicz, "Sliding Mode Control”, Published byJaneza Trdine 9, 51000 Rijeka, Croatia, Copyright (C2011.

[7] W. Perruquetti, J. Pierre Barbot, “sliding mode control in engineering”, (c) 2002 by Marcel Dekker, NEW YORK.

[8] C. Pukdeboon, Alan S. I. Zinober, and May-Win L., "Quasi-Continuous Higher Order Sliding-Mode Controllers for Spacecraft-Attitude-Tracking Maneuvers”, IEEE transactions on industrial electronics, Vol. 57, No. 4, April 2010.

[9] M. DAL, R. Teodorescu, "Sliding mode controller gain adaptation and chattering reduction techniques for DSPbased PM DC motor drives.”, Turk J Elec. Eng \& Comp Sci., Vol.19, No.4, 201.

[10] P.C. Hughes, Spacecraft Attitude Dynamics, John Wiley \& Sons, NewYork, 1986. 